\title{
Optical Signatures of Spin-Orbit Interaction Effects in a Parabolic Quantum Dot
}

\author{
Tapash Chakraborty*‡ and Pekka Pietiläinen ${ }^{* \dagger}$ \\ * Department of Physics and Astronomy, University of Manitoba, Winnipeg, Canada R3T 2N2 and \\ ${ }^{\dagger}$ Department of Physical Sciences/Theoretical Physics, \\ P.O. Box 3000, FIN-90014 University of Oulu, Finland
}

(Dated: October 7, 2018)

\begin{abstract}
We demonstrate here that the dipole-allowed optical absorption spectrum of a parabolic quantum dot subjected to an external magnetic field reflects the inter-electron interaction effects when the spin-orbit interaction is also taken into account. We have investigated the energy spectra and the dipole-allowed transition energies for up to four interacting electrons parabolically confined, and have uncovered several novel features in those spectra that are solely due to the SO interaction.
\end{abstract}

PACS numbers: 71.70.Ej,72.25.Dc,72.25.-b

Far-infrared (FIR) optical absorption spectrum of quantum dots, in particular, dots with a parabolic confinement, has a long and colorful history [1, 2, 3, 4, 5]. First of all, there is a very good theoretical and experimental understanding of the single-electron states in a quantum dot with parabolic confinement. The solution of the Schrödinger equation for an electron confined by a harmonic potential, $v_{c}=\frac{1}{2} m^{*} \omega_{0} r^{2}$ in the presence of an external magnetic field, is well established [2, 6]. The eigenvalues in this case are given by

$$
E_{n l}=(2 n+|l|+1) \hbar \Omega-\frac{1}{2} l \hbar \omega_{c}
$$

where $n, l$ are the principal and azimuthal quantum numbers respectively, $\Omega^{2}=\left[\omega_{0}^{2}+\frac{1}{4} \omega_{c}^{2}\right]$, and $\omega_{c}$ is the cyclotron frequency. Dipole-allowed transitions among these energy levels will have energies [2, 3, 4]

$$
\Delta E_{ \pm}=\hbar \Omega \pm \frac{1}{2} \hbar \omega_{c} .
$$

This relation has been verified to great accuracy by a variety of experiments 1, 2, 5]. Interestingly, however, the observed magnetic field dependent FIR absorption in quantum dots with more than one electron was found to be essentially independent of the number of confined electrons and instead was dominated by the above relation for $\Delta E_{ \pm}$[3]. It was a rather puzzling result because according to this, magneto-optics was clearly incapable of providing any relevant information about the effect of mutual interactions of the confined electrons. The puzzle was later resolved by Maksym and Chakraborty [2, 4], who pointed out that for a parabolic QD in an external magnetic field, the dipole interaction is a function of the center-of-mass (CM) coordinate alone, and only in a parabolic confinement the CM terms of the Hamiltonian are separable. Since the interaction terms are functions of relative coordinates, the observed absorption frequencies are independent of the number of electrons in the dot. Despite this somewhat disappointing performance of a parabolic dot, FIR spectroscopy of QDs (parabolic or otherwise) has generated enormous interest for over a decade that is yet to subside [5]. In this paper we demonstrate that, in the presence of spin-orbit coupling the situation changes considerably. The energy spectra of a parabolic quantum dot containing up to four interacting electrons exhibit structures that are solely due to the presence of SO interaction. Further, the optical absorption spectra of the spin-orbit coupled QDs, reported here for the first time, also exhibit novel features that are direct reflections of the SO coupling effects.

Interest on the role of the spin-orbit coupling in nanostructured systems is now at its peak, due largely to its relevance to spin transport in low-dimensional electron channels [7]. Coherent manipulation of electron spins in low-dimensional systems, in particular in quantum dots, is also expected to pave the way for future electronic and information processing, especially quantum computing and quantum communication [8]. A recent report on the optical detection of spin current in an electron channel [9] due entirely to the electric field is believed to be a major step in that direction. However, most of the experiments reported as yet have used magnetotransport measurements [10] to gain insight about the spin-orbit coupling effects in nanostructures. We propose here that magneto-optical experiments can also be a very useful alternative in that pursuit. The spin-orbit interaction that we are here concerned with is described by the Hamiltonian 11, 12]

$$
\mathcal{H}_{\mathrm{SO}}=\alpha(\vec{k} \times \vec{\sigma})_{z}=\mathrm{i} \alpha\left(\sigma_{y} \frac{\partial}{\partial x}-\sigma_{x} \frac{\partial}{\partial y}\right) .
$$

Here the $z$ axis is chosen perpendicular to the 2DEG (in the $x y$-plane), $\alpha$ is the spin-orbit coupling constant, which is sample dependent and is proportional to the interface electric field, $\vec{\sigma}=\left(\sigma_{x}, \sigma_{y}, \sigma_{z}\right)$ denotes the Pauli spin matrices, and $\vec{k}$ is the planar wave vector. In this potential, the spin of finite-momentum electrons feels a magnetic field perpendicular to the electron momentum in the inversion plane. This results in an isotropic spin splitting energy $\Delta_{\text {SO }}$ at $B=0$ that is proportional to $k$ 7, 11.

Several experimental groups 10 investigating the Shubnikov-de Haas (SdH) oscillations in a 2DEG confined at the heterojunctions with a narrow-gap well (e.g., InGaAs/InAlAs, InAs/GaSb, etc.) have already established that lifting of spin degeneracy results from inver- 
sion asymmetry of the structure which invokes an electric field perpendicular to the layer. Experimentally observed values of the SO coupling strength $\alpha$ lie in the range of 5 - $45 \mathrm{meV} \mathrm{nm} \mathrm{[10].} \mathrm{Energy} \mathrm{levels} \mathrm{of} \mathrm{two} \mathrm{interacting} \mathrm{elec-}$ trons confined in a parabolic quantum dot in an external magnetic field were recently reported by us for this range of SO coupling strength [12]. In the absence of the SO coupling, electron-electron interaction causes the ground state energy to jump from one angular momentum value to another as the magnetic field is increased [2, 4]. The influence of the SO coupling is primarily to move the energy level crossings to weaker fields [12].

The reason why dipole-allowed transitions in a parabolically confined quantum dot can be very different in the presence of $\mathrm{SO}$ interaction is explained as follows. When subjected to the radiation field with amplitude $a$ and polarization $\vec{\epsilon}$, the vector potential $\vec{A}$ in the single particle Hamiltonian

$$
\begin{aligned}
\mathcal{H}_{0}= & \frac{1}{2 m}\left(\vec{p}-\frac{e}{c} \vec{A}\right)^{2}+\frac{1}{2} m \omega_{0}^{2} r^{2} \\
& +\frac{\alpha}{\hbar}\left[\vec{\sigma} \times\left(\vec{p}-\frac{e}{c} \vec{A}\right)\right]_{z}+\frac{1}{2} g \mu_{B} B \sigma_{z}
\end{aligned}
$$

must be replaced with the potential

$$
\vec{A} \rightarrow \vec{A}+\vec{A}_{\omega}, \vec{A}_{\omega}=\vec{\epsilon} a e^{i \vec{k} \cdot \vec{r}-i \omega t} .
$$

In the dipole approximation we assume that

$$
A_{\omega} \approx \vec{\epsilon} a e^{-i \omega t}
$$

and correspondingly the Hamiltonian will be [13]

$$
\mathcal{H} \approx \mathcal{H}_{0}-\mathcal{H}^{\prime} e^{-i \omega t},
$$

where

$$
\mathcal{H}^{\prime}=\frac{e a}{m c} \vec{\epsilon} \cdot\left(\vec{p}-\frac{e}{c} \vec{A}\right)+\frac{\alpha e a}{\hbar c}[\vec{\sigma} \times \vec{\epsilon}]_{z} .
$$

In a many-body system when $\alpha=0$ the first term generates the $\mathrm{CM}$ density excitations where mutual interactions play no role. Consequently (in dipole approximation) only transitions between these modes are possible. When $\alpha$ is different from zero, the second term $\left(\propto \sigma_{x} \epsilon_{y}-\sigma_{y} \epsilon_{x}\right)$ in $\mathcal{H}^{\prime}$ can create spin-density oscillations and interactions have effect on their properties. It is to be noted that, in SO coupled systems the dipole operator still retains its familiar form, $\hat{Q}=\frac{e a}{c} \vec{\epsilon} \cdot \vec{r}$, as is easily verified by evaluating its commutator with the Hamiltonian $\mathcal{H}_{0}$

$$
\left[\hat{Q}, \mathcal{H}_{0}\right]=i \hbar \mathcal{H}^{\prime} .
$$

Dipole operator is independent of the electron spin. The dipole-allowed optical transitions are always from the same spin states, but the angular momenta must differ by unity. In the presence of SO coupling, neither the dipole operator nor the selection rule changes, but the SO interaction mixes the neighboring angular momentum values $(l$ and $l+1)$ as well as the spin and hence the selection rule now applies to the total angular momentum $J$ as well. Therefore, transitions from other states that are not allowed without the SO coupling, are now allowed.

For numerical evaluation of the optical absorption spectrum, we have considered a InAs quantum dot where most of the spin-related phenomena have been studied [10]. We have considered up to four interacting electrons in the quantum dot. Evaluation of the transition energies of a quantum dot containing more than two electrons is quite challenging. Firstly, since we work in the occupation space spanned by direct anitisymmetrized products of the spinors

$$
\left|\lambda_{l}\right\rangle=\left(\begin{array}{c}
\sum_{n=0} u_{n}^{\lambda_{l}} f_{n l} e^{i l \theta} \\
\sum_{n=0} d_{n}^{\lambda_{l}} f_{n, l+1} e^{i(l+1) \theta}
\end{array}\right),
$$

where $f_{n l}=\sqrt{n ! /(n+|l|) !} x^{|l| / 2} e^{-x / 2} L_{n}^{l}(x), u_{n}^{\lambda_{l}}$ and $d_{n}^{\lambda_{l}}$ are the expansion coefficients [12], the two-body matrix elements are composed of Coulomb matrix elements expressed in a parabolic quantum dot as finite sums [2]. In this case however, the expansions can extend to Laguerre polynomials of large degree and large angular momenta which leads to well known numerical instabilities [14]. To avoid these, we had to resort to multiple precision

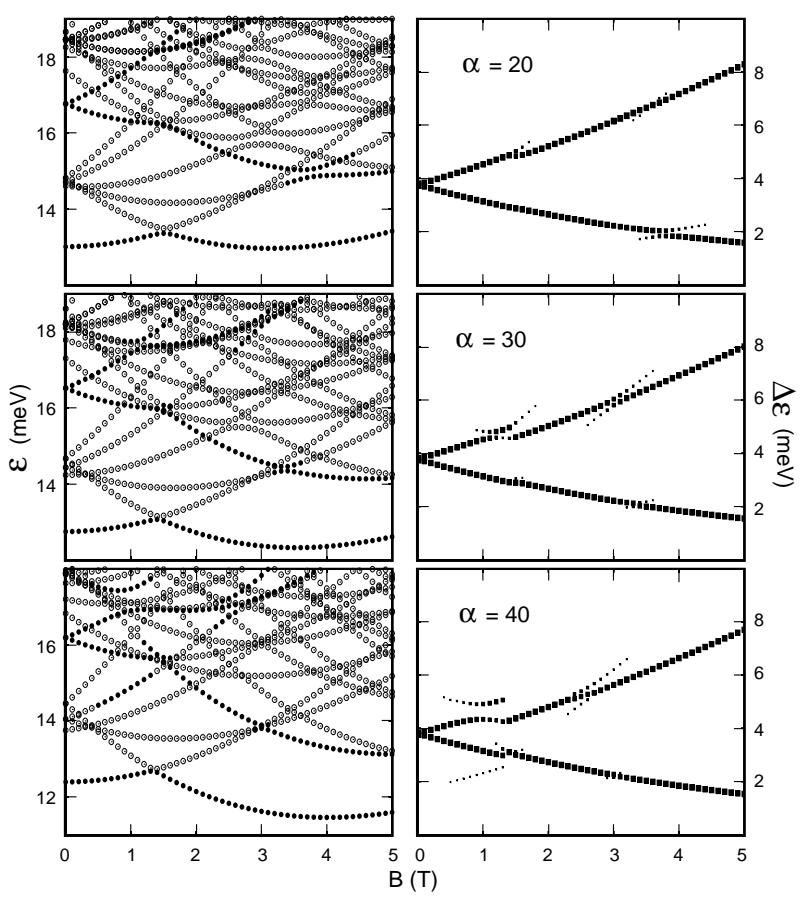

FIG. 1: Energy spectra (left panel) and dipole-allowed transition energies (right panel) for two interacting electrons confined in a InAs quantum dot and for various values of the SO coupling strength $\alpha[\mathrm{meV} \mathrm{nm}]$. The solid dots in the energy spectrum identifies the energy levels involved in transitions that correspond to the lowest branches of the absorption spectra (in the right panel). In the right panels, the size of the points in the figures is proportional to the calculated intensity. 
arithmetics (but, in order to circumvent the resulting extremely long computation times, we had to tabulate selected subsummations). Secondly, since in the presence of the spin-orbit interaction all possible spin configurations need to be considered, the dimension of the Hilbert space required for convergence can easily become huge (of the order of $10^{6}$ for four electrons). To diagonalize these "monster matrices" we implemented the DavidsonLiu algorithm [15] that is very suitable for this task.

Our numerical results for energy spectra and absorption spectra (dipole-allowed) are presented in Figs. 1-3, for 2-4 electrons and for various values of the SO coupling strength $\alpha$. We have considered the following parameters for the InAs quantum dot: $m^{*} / m_{0}=0.042, \epsilon=14.6, g=$ -14 and $\hbar \omega_{0}=3.75 \mathrm{meV}$. The dipole matrix elements are evaluated from

$$
\begin{aligned}
d_{\lambda_{1} \lambda_{2}}= & \left\langle\lambda_{1}\left|r e^{i \theta}\right| \lambda_{2}\right\rangle \\
= & \delta_{l_{1}, l_{2}+1} \sum_{n}\left[\sqrt{n+l_{1}} u_{n}^{\lambda_{1}} u_{n}^{\lambda_{2}}-\sqrt{n} u_{n-1}^{\lambda_{1}} u_{n}^{\lambda_{2}}\right. \\
& \left.+\sqrt{n+l_{1}+1} d_{n}^{\lambda_{1}} d_{n}^{\lambda_{2}}-\sqrt{n} d_{n-1}^{\lambda_{1}} d_{n}^{\lambda_{2}}\right]
\end{aligned}
$$

when $l_{1} \geq 0$ and a similar type of equation for $l_{1}<$ 0 . The intensity is obtained from $\mathcal{I} \propto\left|d_{\lambda_{1} \lambda_{2}}\right|^{2}[16]$. In our plots for the absorption spectra (the right panels of the figures), the size of the points is proportional to the calculated intensity.

A striking feature visible in the absorption spectra (right hand panels of the figures) is the appearance of discontinuities, anticrossings and new modes in addition

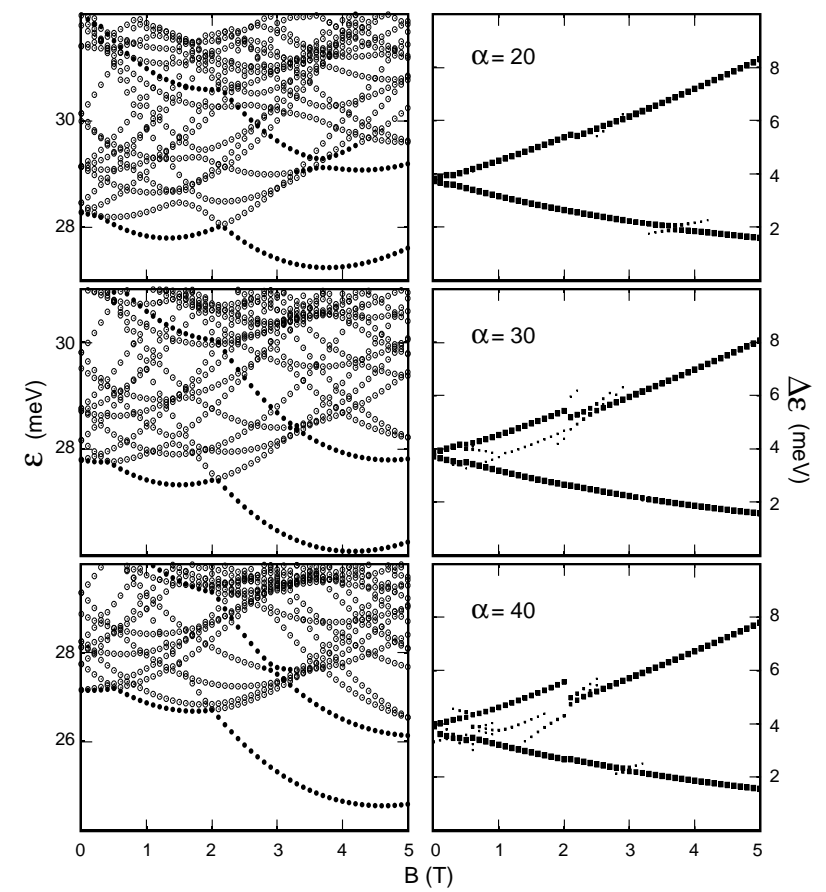

FIG. 2: Same as in Fig. 1, but for three interacting electrons in a quantum dot. to the two main $(\alpha=0)$ absorption lines. These optical signatures of the SO interaction are consequences of the multitude of level crossings and level repulsions that occur in the energy spectra (left hand panels of Figs. 1-3). The latter ones can be attributed to an interplay between $\mathrm{SO}$ and Zeeman couplings. In order to understand their origin, let us first examine the case of the two electron system. In our spinor notation the main contribution to the ground state at zero magnetic field comes from the two-electron state $\left|\lambda_{l_{1}}, \lambda_{l_{2}}\right\rangle=\left|\lambda_{0}, \lambda_{-1}\right\rangle$, where $\left|\lambda_{l_{1}}\right\rangle$ is a spinor with $J_{1}=l_{1}+1 / 2=1 / 2, d_{n}^{\lambda_{1}}=0$, and $\left|\lambda_{l_{2}}\right\rangle$ a spinor with $J_{2}=-1 / 2$ and $u_{n}^{\lambda_{2}}=0$, i.e., both electrons have zero orbital angular momenta with opposite spins (corresponding to $J=J_{1}+J_{2}=0$ ). Now when we increase the magnetic field the spin triplet configuration will become, due to the interaction, energetically more favorable. If the Lande factor is negative then the electrons would like to occupy states with orbital angular momenta 0 and -1 with both spins up. In the spinor picture this means that $\left|\lambda_{l_{2}}\right\rangle$ still has $l_{2}=-1(J=0)$ but now $u_{n}^{\lambda_{2}} \neq 0$ and $d_{n}^{\lambda_{2}}=0$. The SO interaction mixes these two configurations which results in a level repulsion. On the other hand, when the strength of the SO coupling is further increased, the relative significance of the Zeeman contribution to $\mathcal{H}_{0}$ decreases. The energy shifts to states with $J \neq 0$ will then become energetically feasible and we have again crossings of levels. For increasing number of electrons in the dot, the energy spectra is more dense and exhibit additional level crossings [Figs. 2-3]. As a consequence, the ground state momentum also changes

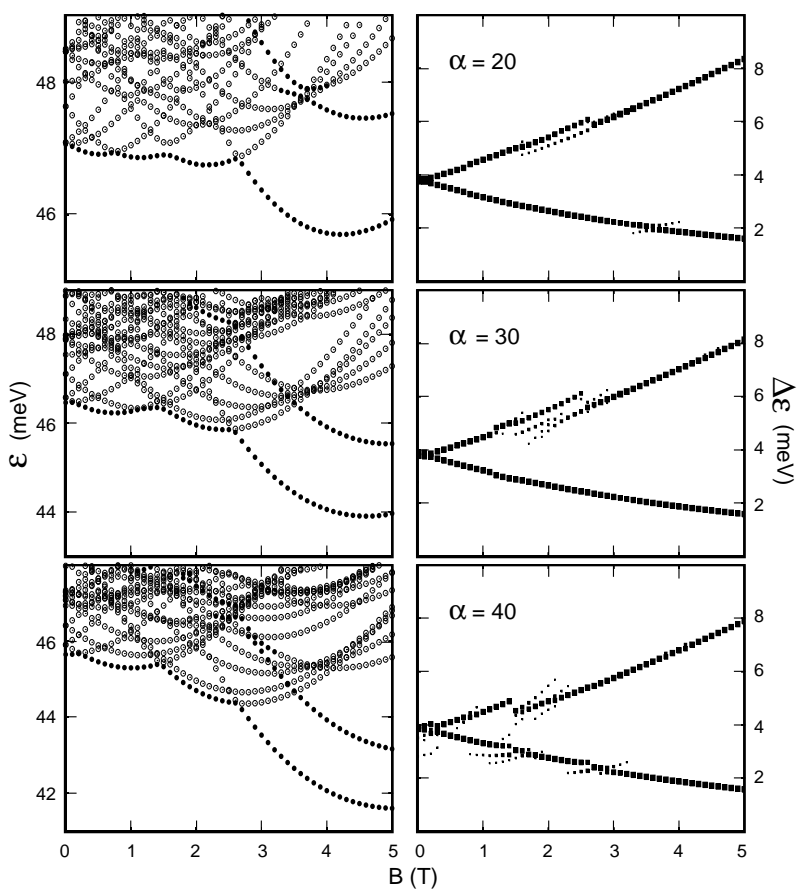

FIG. 3: Same as in Fig. 1, but for four interacting electrons in a quantum dot. 
more frequently as compared to that of the two electron case.

At moderate SO coupling strengths the absorption spectra do not essentially differ from the single particle spectrum. But when the coupling strength increases the deviation from the pure parabolic confinement also increases which in turn implies that the lowest final states of dipole allowed transitions are not any more achievable by adding $\hbar \Omega \pm \frac{1}{2} \hbar \omega_{c}$ to the initial state energies. In particular, this results in discontinuities and anticrossing behaviors as well as appearance of new modes. As an illustration, let us consider the absorptions that at the magnetic field $B=1 \mathrm{~T}$ take the two electron system from the ground state to excited states. In the absence of the SO coupling the ground state is a spin singlet state $S=0$ with total angular momentum $J=0$. According to the dipole selection rules absorptions cause transitions to states $J= \pm 1$ and $S=0$ with energies $\Delta E_{ \pm}$above the ground state. Looking at the bottom right panel of Fig. 1, we note that in addition to the two main lines there are now two additional lines (at $B=1$ $\mathrm{T}$ ) of appreciable intensity at the $\mathrm{SO}$ coupling strength $\alpha=40$. Further analysis reveals that the ground states still have $J=0$ and that the expectation value of the spin $z$-component is $\left\langle\sigma_{z}\right\rangle=0$. The excited states also have $J= \pm 1$, as before. However, the final spin states can no longer be classified as singlets: the expectation values $\left\langle\sigma_{z}\right\rangle$ vary between -0.03 and 0.39 . When the number of electrons increases the number of these additional modes also increases but at the same time the relative intensities decrease (at each $B$ we have normalized the total intensity to unity). On the other hand, the discontinuities as consequences of deviations from a parabolic confinement become more pronounced (Figs. 2-3).

In conclusion, we have presented the energy levels and optical absorption spectra of up to four interacting electrons in a parabolic quantum dot subjected to an externally applied perpendicular magnetic field. The optical absorption spectra exhibit new modes that are a direct consequence of the SO coupling effects. These are manifested in the energy spectra as multiple level crossings and level repulsions that are attributed to an interplay between the Zeeman and SO couplings present in the system Hamiltonian.

We would like to thank Marco Califano for many helpful discussions. The work of T.C. has been supported by the Canada Research Chair Program and the Canadian Foundation for Innovation (CFI) Grant.
[†] Electronic mail: tapash@physics.umanitoba.ca

[1] T. Chakraborty, Comments Condens. Matter Phys. 16, 35 (1992); V. Gudmundsson, A. Manolescu, R. Krahne, and D. Heitmann, in Nano-Physics \& Bio-Electronics: A New Odyssey, Edited by T. Chakraborty, F. Peeters, and U. Sivan (Elsevier, 2002).

[2] T. Chakraborty, Quantum Dots (North-Holland, Amsterdam, 1999).

[3] C. Sikorski and U. Merkt, Phys. Rev. Lett. 62, 2164 (1989).

[4] P.A. Maksym and T. Chakraborty, Phys. Rev. Lett. 65, 108 (1990).

[5] See, for example, I. Magnusdottir and V. Gudmundsson, Phys. Rev. B 60, 16591 (1999); H. Imamura, P.A. Maksym, and H. Aoki, Phys. Rev. B 53, 12613 (1996); 59, 5817 (1999); R. Krahne, V. Gudmundsson, C. Heyn, and D. Heitmann, ibid. 63, 195303 (2001); E. Lipparini, N. Barberan, M. Barranco, M. Pi, and L. Serra, ibid. 56, 12375 (1997); P.A. Maksym, Physica B 184, 385 (1993); T. Seki, Y. Kuramoto, and T. Nishino, J. Phys. Soc. Jpn. 65, 3945 (1996); R. Ugajin, Phys. Rev. 51, 10714, 11136 (1995); M. Wagner, A.V. Chaplik, and U. Merkt, ibid. 51, 13817 (1995); B. Meurer, D. Heitmann, and K. Ploog, Phys. Rev. Lett. 68, 1371 (1992); T. Chakraborty, V. Halonen, and P. Pietiläinen, Phys. Rev. B 43, 14289 (1991); T. Demel, D. Heitmann, P. Grambow, and K. Ploog, Phys. Rev. Lett. 64, 788 (1990); A. Lorke, J.P. Kotthaus, and K. Ploog, ibid., 64, 2555 (1990).

[6] V. Fock, Z. Phys. 47, 446 (1928); C.G. Darwin, Proc. Cambridge Philos. Soc. 27, 86 (1930).

[7] D.D. Awschalom, D. Loss, and N. Samarth (Eds.), Semiconductor Spintrinics and Quantum Computation
(Springer, 2002); D.D. Awschalom, R.A. Buhrman, J.M. Daughton, S von Molnar, and M.L. Roukes (Eds.), Spin Electronics (Kluwer, 2004); D. Grundler, Phys. World 15, 39 (2002); S.A. Wolf, et al., Science 294, 1488 (2001); G.A. Prinz, Phys. Today 48, 58 (1995).

[8] D. Loss, G. Burkard, and D.P. DiVincenzo, J. Nanoparticle Res. 2, 401 (2000).

[9] Y.K. Kato, R.C. Myers, A.C. Gossard, and D.D. Awschalom, Science 306, 1910 (2004).

[10] D. Grundler, Phys. Rev. Lett. 84, 6074 (2000); C.-M. Hu, J. Nitta, T. Akazaki, H. Takayanagi, J. Osaka, P. Pfeffer and W. Zawadzki, Physica E 6, 767 (2000); C.-M. Hu, et al., Phys. Rev. B 60, 7736 (1999); Y. Sato, T. Kita, S. Gozu, and S. Yamada, J. Appl. Phys. 89, 8017 (2001); T. Matsuyama, C.-M. Hu, D. Grundler, G. Meier, and U. Merkt, Phys. Rev. B 65, 155322 (2002); J. Nitta, T. Akazaki, and H. Takayanagi, Phys. Rev. Lett. 78, 1335 (1997); T. Koga, J. Nitta and S. Datta, Physica E 18, $161(2003)$.

[11] Y.A. Bychkov and E.I. Rashba, J. Phys. C 17, 6039 (1984).

[12] T. Chakraborty and P. Pietiläinen, cond-mat/0410248 (to be published).

[13] P. Tonello and E. Lipparini, Phys. Rev. B70, 081201 (2004).

[14] M. Stone, H.W. Wyld, and R.L. Schult, Phys. Rev. B 45, 14156 (1992).

[15] E.R. Davidson, Computers in Physics 7, 519 (1993).

[16] V. Halonen, P. Pietiläinen, and T. Chakraborty, Europhys. Lett. 33, 377 (1996). 